\title{
Induction and transmission of oncogene-induced senescence
}

\author{
Nattaphong Rattanavirotkul ${ }^{1} \cdot$ Kristina Kirschner $^{2} \cdot$ Tamir Chandra $^{3}$ (D)
}

Received: 1 February 2020 / Revised: 27 July 2020 / Accepted: 3 September 2020 / Published online: 16 September 2020 (c) The Author(s) 2020

\begin{abstract}
Senescence is a cellular stress response triggered by diverse stressors, including oncogene activation, where it serves as a bona-fide tumour suppressor mechanism. Senescence can be transmitted to neighbouring cells, known as paracrine secondary senescence. Secondary senescence was initially described as a paracrine mechanism, but recent evidence suggests a more complex scenario involving juxtacrine communication between cells. In addition, single-cell studies described differences between primary and secondary senescent end-points, which have thus far not been considered functionally distinct. Here we discuss emerging concepts in senescence transmission and heterogeneity in primary and secondary senescence on a cellular and organ level.
\end{abstract}

Keywords Oncogene-induced senescence $\cdot$ Secondary senescence $\cdot$ Notch signalling $\cdot$ Juxtacrine senescence

\section{Introduction}

Cellular senescence is a stress response programme that irreversibly and stably arrests the cell cycle following a variety of intrinsic and extrinsic stressors such as shortening of the chromosomal termini, DNA damage, oxidative stress, oncogenic and mitogenic stimuli [1-3]. Cellular senescence was first described by Hayflick and Moorhead, who observed that cultured human fibroblasts undergo a finite number of cell divisions, a phenomenon later widely known as the 'Hayflick limit' and termed as replicative senescence [4]. The limited replicative capacity of cells in vitro spontaneously suggests a model for ageing at the cellular level. It is now clear that

Nattaphong Rattanavirotkul

nattaphong.rat@mahidol.ac.th

$\triangle$ Kristina Kirschner

kristina.kirschner@glasgow.ac.uk

Tamir Chandra

tamir.chandra@igmm.ed.ac.uk

1 Chakri Naruebodindra Medical Institute, Ramathibodi Medical School, Faculty of Medicine Ramathibodi Hospital, Mahidol University, 111, Bang Pla, Bang Phli, Samut Prakan 10540, Thailand

2 Institute of Cancer Sciences, University of Glasgow, Glasgow G61 1BD, UK

3 MRC Human Genetics Unit, MRC Institute of Genetics and Molecular Medicine, The University of Edinburgh, Edinburgh, UK this proliferative arrest is mainly driven by telomere attrition [5]. Initially dismissed as an in vitro artefact that bore no relevance to biological mechanisms in vivo, the concept of cellular senescence has stood the test of the past five decades and evolved to become relevant beyond the ageing context, with critical physiological functions in preventing tumour formation, remodelling of tissues during embryonic development, and promoting wound healing and immunogenic clearance [6].

In the replicative senescence model of proliferating human cells, successive telomere erosion in each cell division ultimately uncaps the free double-stranded chromosome ends, causing them to be recognised as DNA double-strand breaks (DSBs) and activating the DNA damage response (DDR) pathway [7, 8]. The telomere dysfunction-initiated DDR signalling engages the ataxia telangiectasia-mutated (ATM) kinase that subsequently stabilises the tumour suppressor protein 53 (TP53), leading to an upregulation of its target the cyclin-dependent kinase (Cdk) inhibitor (CDKN1A). The TP53/CDKN1A pathway implements a cell cycle exit by inhibiting the activity of cyclin E-CDK2 and allowing the hypophosphorylated form of the retinoblastoma (RB) tumour suppressor to enable G1 arrest [9]. Alternatively, senescence can be established independently of the TP53/CDKN1A axis through the p16/RB tumour suppressor pathway. The cyclin dependent kinase inhibitor $2 \mathrm{~A}$ (CDKN2A) locus encodes p16 and ARF, with the former 
repressing CDK4/6 while the latter cross-talking with the TP53/CDKN1A network.

Unlike quiescent or terminally differentiated cells, senescent cells do not respond to mitogenic cues and are not specialised cells resulting from a developmentally differentiated program. Senescent cells display distinguishing characteristics morphologically and biochemically. They are generally characterised by an enlarged and flattened shape and robustly express the lysosomal enzyme $\beta$-galactosidase, detectable by senescence-associated b-galactosidase (SA-b-gal) staining at sub-optimal pH [10]. Increased levels of p16 are also commonly used as a marker of senescence in vitro and in vivo. Another prominent feature is the formation of senescenceassociated heterochromatic foci (SAHF), a rearrangement of heterochromatin into discrete nuclear subdomains visible by 4',6-diamidino-2-phenylindole (DAPI) staining. One of the most striking changes associated with senescence is the secretion of a suite of pro-inflammatory cytokines and chemokines, extracellular matrix proteins, growth factors and metalloproteinases as part of a senescence-associated secretory phenotype (SASP) [11]. SASP components attract immune cells for clearance of senescent cells, ensuring tissue homeostasis. Another emerging fundamental feature of senescent cells is accumulation of lipofuscin, insoluble granules formed from damaged and cross-linked proteins [12]. Lipofuscin accumulation reflects macromolecular damage and altered metabolism, both of which have been reported as hallmarks of senescence, and has been exploited for identification of senescence in vitro and vivo [13, 14] as well as proposed as the first screening step in a multimarker algorithmic approach for senescent cell detection [15]. However, to date, none of the senescence markers are absolutely reliable or specific for the in vivo identification of all senescence types and different markers must be used in combination to conclusively recognise senescent cells. Discovering novel senescence biomarkers that are of universally biological interest is, therefore, an ongoing quest in the field. Overall, the most recent consensus from the International Cell Senescence Association (ICSA) summarises the four interdependent hallmarks of senescence as: (1) cell cycle arrest; (2) SASP; (3) macromolecular damage; and (4) deregulated metabolism [15].

\section{Oncogene-induced senescence: primary senescence}

Among a plethora of senescence stimuli, intrinsic changes from activated oncogenes invoke not only an irreversible state of proliferative arrest but also a biologically meaningful response that curbs malignant transformation. This mode of senescence is referred to as oncogene-induced senescence (OIS), also considered as primary senescence. The first observation of OIS was described when an oncogenic form of Ras, $\mathrm{Hras}^{V 12}$, was ectopically expressed in primary cell culture of human lung fibroblasts IMR90. Senescent growth arrest is also stably established in vivo with a notable example being the ectopic expression of Ras in mammary epithelial cells [16]. In contrast to replicative senescence, OIS occurs in a telomere erosion-independent manner and can engage both the TP53/CDKN1A and p16/RB networks. Other studies have shown that loss of TP53 or its regulator p19 in mice provokes Ras-induced cancer cell invasion, while the reactivation of TP53 suppresses tumour growth whilst displaying common senescence markers [17-19]. OIS cells commonly have increased expression of p16 and can escape from senescence and re-enter the cell cycle in the event of low p16 levels [20]. The maintenance of OIS is dependent upon the accumulation of RB, which acts downstream of p16 through repression of E2F-target genes associated with DNA replication [21]. Despite much evidence on the well-established involvement of TP53 and p16 in OIS, in certain cell types and contexts TP53 and p16 are not required for the initiation and maintenance of OIS [22, 23]. Discordant results were also reported in human mammary epithelial cells, which undergo Ras-induced senescence without engaging p16 and TP53 [24]. This seems to reflect the variability between cell types in undergoing TP53- or p16-mediated senescence.

Recent studies have implicated the NOTCH signalling pathway as one of the regulators of OIS. NOTCH signalling is an evolutionarily conserved pathway and was first described in cell-fate decisions during wing development in Drosophila [25]. The canonical NOTCH pathway relies on a NOTCH receptor expressed on the signal-receiving cell upon direct contact with its ligand on the adjacent signal sending cell. This ligand-receptor interaction eventually leads to cleavage of the NOTCH intracellular domain (NICD), a constitutively active form of $\mathrm{NOTCH}$, which enters the nucleus to bind to a transcriptional complex containing Mastermind-like 1 (MAML1) and other factors to activate target gene expression. Network enrichment analysis and flow cytometry have revealed upregulation of NOTCH1 in OIS cells accompanied by induction of transforming growth factor-beta (TGF $\beta$ ) [21]. However, the increased NOTCH activity during OIS was only transient while cells transition into the early phase of primary OIS. The latter phase of OIS establishment saw the return of N1ICD and NOTCH-1 target levels to resting activity. Strikingly, similar to oncogenic activation, ectopic expression N1ICD was shown to drive cell-autonomous senescence, in what is known as Notchinduced senescence (NIS) or primary NIS, in human fibroblasts with a distinct secretory phenotype and chromatin structure from that of primary OIS triggered by Ras activation [26, 27]. For example, primary NIS cells show a reduction of basal levels of the proinflammatory cytokine IL-8 and activation of a canonical NOTCH ligand, JAG1, as well as TGF $\beta 1$, while lacking SAHF formation [26, 27]. Overall, 
these pioneering studies of NIS clearly demonstrated the contribution of NOTCH signalling as a transient component of primary senescence. Given the diverse roles of NOTCH signalling in development, cell differentiation and cancer, it is of fundamental interest to investigate the functional significance of NOTCH in senescence, which remains to be established.

The physiological role of primary OIS remains clear and unequivocal: serving as an early, cell-intrinsic tumoursuppressive mechanism. Multiple studies in lung and pancreas have shown that conditional activation of oncogenic $\mathrm{KRAS}^{\mathrm{V} 12}$ led to neoplastic transformation in pre-malignant conditions that were accompanied by senescence markers [28]. Another in vivo study in mouse lymphoid cells demonstrated that NRAS overexpression triggered OIS that prevents full lymphoma progression [29]. The tumoursuppressive role of OIS is also evident in the mouse prostate following depletion of the tumour suppressor PTEN, but not when both PTEN and TP53 were lost in advanced prostate cancer [30]. Crucially, in vitro and in vivo experiments in human melanocytic naevi (moles) present one of the most intriguing findings showing OIS as a barrier to tumourigenesis [31]. Up to $80 \%$ of these naevi harbour the $B R A F^{V 600 E}$ mutation, the most frequent mutation in melanomas. $B R A F^{V 600 E}$-expressing melanocytes persist in the growth-arrested state and display several OIS markers such as upregulation of $\mathrm{p} 16$ and positive SA- $\beta$-gal staining [32, 33]. Still, they retain the ability to transform into malignancy if BRAF-induced senescence is silenced or reversed. Naevi are therefore benign lesions, that arrest stably for decades before the course of melanogenesis takes place, representing one of the best available examples of OIS in vivo. It is therefore of great biological interest to understand the tumour suppressor state and its genomic dynamics before cells escape from senescence and progress into full-blown melanoma. Examples of in vivo OIS are numerous and have been extensively reviewed elsewhere $[34,35]$.

\section{Non-cell-autonomous aspects of OIS (secondary senescence)}

\section{Paracrine transmission of senescence}

Paradoxically, the past few years have witnessed contentious challenges to the prevailing function and mechanism of OIS, revealing more diverse and sometimes contradictory roles of OIS than previously believed. Far from being a simple and terminal state of replicative cessation, OIS cells communicate and interact with each other, consistent with the fact that OIS cells remain metabolically active. Pioneering studies showed that OIS cells are able to self-amplify expression of their SASP components such as Intereukin
6/8 (IL-6 and IL-8) alongside other inflammatory molecules in an autocrine manner, by reinforced activation of nuclear factor kappa-light-chain-enhancer of activated B cells (NF$\kappa \mathrm{B})$ and CCAAT-enhancer-binding protein beta (C/EBP $\beta)$ $[36,37]$. Subsequent work then described the ability of senescent cells to engage in cell-to-cell communication, modifying their microenvironment via paracrine signalling and establishing a mode of senescence named secondary senescence [38]. When co-culturing OIS and proliferating cells, OIS cells induced a senescence phenotype in their growing neighbouring cells through locally secreted SASP molecules [38]. Quantitative proteomics and small-molecule inhibitor screens have identified complex inflammasomes and secretomes containing transforming growth factor beta (TGF $\beta)$, vascular endothelial growth factor (VEGF) and chemokine CC motif ligand 2 (CCL2) as modulators of paracrine senescence. Interleukin 1 (IL-1) signalling was further shown to be a key upstream regulator of the proinflammatory signalling cascade and inhibition of the NLR family pyrin domain containing 3 (NLRP3) inflammasome, which regulates IL- $1 \beta$, led to the suppression of the SASP phenotype [38]. Additional findings have also pointed to the interplay between DNA damage, oxidative stress and paracrine senescence mediated by the release of IL- 1 and TGF $\beta$, with DDR signalling being shown to induce senescence in bystander cells [39].

The SASP secretome, as a mediator of paracrine activities of OIS, displays complex and dynamic features, part of which are regulated by NOTCH signalling [Fig. 1]. By comparing the transcriptomes and proteomes of NIS fibroblasts to those of the classic primary Ras-induced senescence (RIS), a time-course switch in the SASP-associated secretomes was revealed [26]. High levels of NOTCH1 drove a TGF- $\beta$-dominant secretome in the first wave of OIS secretion and at the same time inhibited a C/EBP $\beta$ orchestrated SASP expression. The second wave was characterised by low levels of NOTCH1 and the reliance on C/ EBP $\beta$-dominant expression of proinflammatory cytokines. These two biochemically distinct and functionally opposing secretomes highlight the heterogeneity of SASP during the progression of OIS and the possible involvement of its spatio-temporal fluctuations in modulating paracrine transmission of senescence. What remain to be determined are the factors that control the switch of SASP dynamics and their physiological relevance.

As paracrine senescence is consistently observed in human and mouse models of OIS [38], the previously unappreciated non-cell-autonomous activities of OIS manifest a critical link between the expression of SASP factors and the induction of paracrine senescence in vivo, with farreaching biological implications in determining the cellular outcome of normal cells in the context of tumourigenesis. By means of secreting SASP factors into the extracellular 


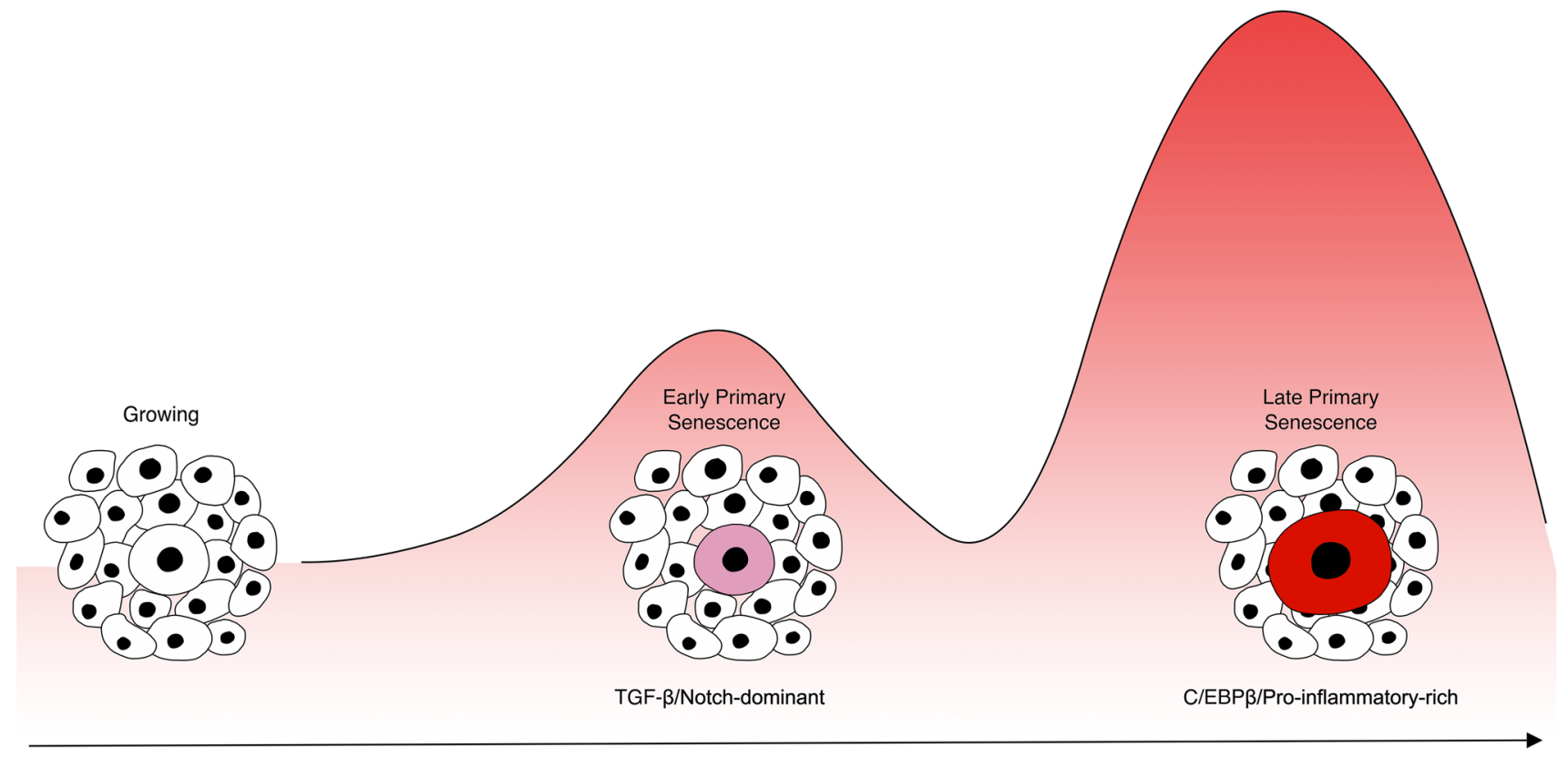

OIS

Fig. 1 Schematic representation of the dynamic progression of primary oncogene-induced senescence. Primary OIS cells display two main phases, each with distinct a distinct SASP profile. The first or

compartment, not only can OIS cells recruit immune cells to eliminate tumour cells $[40,41]$, but they can also promote tumourigenesis when physiological conditions become unfavourable. In skin, prostate and liver cancer models, the SASP phenotype is found to facilitate migration of cancer cells or favour an immunosuppressive microenvironment, which increases the risk of proliferation, angiogenesis and metastasis [42-44]. Taken together, it is becoming increasingly appreciated that OIS possesses a dual role in cancer pathogenesis, and this is largely dependent on the tissue context, the composition of the SASP and the duration of the senescence state. In this respect, there have been attempts to selectively remove non-advantageous senescent cells that may adversely transform their surrounding cells into malignant cells without affecting the functionally beneficial senescent cells. This has proved to be a success in a transgenic mouse system where p16-expressing senescent cells can be selectively eliminated [45]. Clearance of these p16-positive cells improved age-associated phenotypes, lifespan and tissue rejuvenation. A follow-up study then confirmed a significant reduction of spontaneous tumour formation after selective removal of senescent cells [46]. Paradoxically, a very recent report has demonstrated a slow process of senescence accumulation during a lifespan of p16-knock in mice whose p16high hepatic senescence cells appear to have important structural and functional roles [47]. Removal of liver cells with p16high led to liver and perivascular fibrosis, impairing the health of the animals. This suggests that the early stage of primary OIS is characterised by increased TGF $\beta$ signalling and NOTCH-dominant activity. The second or late stage of primary OIS is driven by $\mathrm{C} / \mathrm{EBP} \beta$-rich and pro-inflammatory factors

timing of senescence might be a key factor that determines the outcome of physiological and pathological conditions. Similarly, another observation also showed that C/EBP $\beta$ is a key player in the TGF $\beta$ cytostatic response by regulating expression of $\mathrm{p} 15 \mathrm{Ink} 4 \mathrm{~b}$, which contradicts the notion that OIS phase I involves TGF $\beta$ but not C/EBP $\beta$ [48]. It remains to be tested whether such contradictory results are physiologically relevant with the paradox observed in the elimination of senescent cells.

Hence, the concept of OIS as an in vivo antagonistic pleiotropy has emerged, featuring an anti-cancer action in early stages of oncogenesis and a switch to a deleterious, procancer phase over time with advanced age. The key questions now needing answering are when and why exactly the twisted turn of senescence occurs. Pivotal to understanding the complexity of senescent effects is a comprehensive detail of how senescent cells interact with their microenvironment through short- and long-term secretion of SASP factors and accurate SASP factor composition.

More recently, the paracrine transmission of senescence has been found to play a role in cellular reprogramming, as shown when SASP components induced senescence and encouraged reprogramming by activation of octamer-binding transcription factor 4 (Oct4), SRY-box 2 (Sox2), Kruppel like factor 4 (Klf4) and MYC proto-oncogene, bHLH transcription factor (c-Myc) (OSKM or Yamanaka factors) in non-senescent cells $[49,50]$. These studies collectively serve to improve our understanding of the complex network 
of the SASP, which contains many layers of regulation for signal amplification and exploits paracrine mechanisms to achieve secondary senescence in the cell neighbourhood both in vitro and in vivo. In addition, other modes of non-cell-autonomous activities of senescence have been described, including direct protein transfer through membrane protrusions connecting two cells called cytoplasmic bridges [51] and extracellular vesicles secreted from senescent cells $[52,53]$.

\section{Juxtacrine transmission of senescence}

The molecular basis for controlling secondary senescence and the non-cell-autonomous properties of OIS are still under active investigation and revision, as the concept of secondary senescence is relatively novel and likely to be understood not as a static or permanent process, but as a highly dynamic and variable outcome depending on heterogeneous SASP secretomes. Coming into play is the contribution of NOTCH as a key mediator of contact-dependent secondary senescence, or juxtacrine senescence. Provided that the canonical action of NOTCH in mediating cell-tocell signalling has critical implications in diseases such as cancer, the idea that senescence can spread by NOTCH1 signalling via direct cell-to-cell contact is an appealing notion that has only been pursued recently. As discussed earlier, the role of NOTCH signalling in senescence has been explored in NIS as a primary cell-autonomous process [21]. The same study also further revealed the non-cell-autonomous effects of NIS cells through a cell-contact dependent mechanism that propagates secondary senescence in their surrounding environment. Through ectopic expression of N1ICD, the primary senescent N1ICD-expressing cells were able to mediate lateral transmission of senescence to their neighbouring naïve cells in a JAG1-dependent manner. Inhibition of NOTCH signalling consequently compromised non-cellautonomous secondary senescence. NOTCH-mediated juxtacrine senescence was further shown in a subsequent study to non-autonomously regulate chromatin structure in normal adjacent cells, repressing SAHF formation by suppressing expression of HMGA genes [23].

Despite the clear evidence of NOTCH signalling in facilitating juxtacrine secondary senescence, the nature of the secondary senescence population itself was not explored or characterised. The important question is whether NOTCH acts as a senescence inducer in neighbouring naïve cells that have not experienced oncogenic insults, in addition to SASP, particularly in the context of primary OIS cells that do not undergo ectopic activation of NOTCH. Recent work has presented a conceptual advance that primary and secondary senescence can be viewed as two distinct transcriptional termini and that NOTCH is an important effector of secondary senescence [54] [Fig. 2]. Single-cell RNA sequencing approaches were used to investigate the heterogeneity within OIS and identified two major transcriptional endpoints, one being a primary senescent population and the other a secondary senescent population. In contrast to the traditional view that paracrine senescence is the only mediator of secondary senescence in OIS, our downstream transcriptomic analyses revealed a pronounced enrichment of NIS signature in the secondary senescence population, suggesting NOTCH as a potent mediator. The requirement for $\mathrm{NOTCH}$ signalling for secondary senescence induction was confirmed in vitro through a co-culture system between NOTCH-incompetent cells and primary OIS cells. As opposed to the outcome of pure primary NIS, where the pro-inflammatory SASP components are suppressed and fibrillar collagens induced, the OIS-mediated secondary senescent cells showed both expression of SASP factors and induction of fibrillar collagens. Distinguishing the complex crosstalk between two modes of secondary senescence was only possible at single cell resolution. To further dissect the contribution of NOTCH to the activation of secondary senescence, secondary cells with impaired NOTCH signalling were co-cultured with primary OIS cells, and compared with secondary cells with intact NOTCH. Gene set enrichment analysis confirmed that the NOTCH-compromised cells upregulated the interferon-gamma response and pro-inflammatory factors relative to the normal secondary cells, suggesting that NOTCH signalling blunted the SASP response to a certain extent. Furthermore, this study provided in vivo data in secondary senescent-induced hepatocytes, which showed upregulation of NOTCH signalling compared to primary senescent hepatocytes. This concept was strengthened in an in vivo liver cancer model in which the recruitment of immune cells was compromised by the lateral transmission of NOTCH1mediated senescence [26].

As research on secondary senescence is still in the nascent stage, the exact role and biological significance of NOTCHmediated juxtacrine senescence and secondary senescence, in general, remain elusive. At least, it has become increasingly apparent that throughout the course of OIS, cells mature from pre-senescence to complete senescence, undergoing different stages where secondary senescence signals are dynamically transmitted to adjacent cells. Principally, secondary senescence encompasses both paracrine and juxtacrine modalities, but exact nature and timing of their operation and regulation is just beginning to emerge. In combination with the proposed model of the transient NOTCH/ TGF- $\beta$-dominant secretome in the early phase of OIS and pro-inflammatory C/EBP $\beta$-rich SASP in the late phase, the results from the single cell RNA-sequencing experiment of OIS cells suggest that juxtacrine senescence is predominantly achieved in the early NOTCH phase, while paracrine senescence takes on a more influential role when OIS is fully established $[26,27,54]$. The transition between the two 
Fig. 2 Schematic representation of how secondary senescence is established from primary senescence. There are two forms of primary senescence: oncogene-induced senescence (OIS) and Notch-induced senescence (NIS). These primary senescent cells can further trigger secondary senescence in their neighbouring cells in the process called secondary senescence, which can be mediated by cell-to-cell contact and/ or paracrine transmission of senescence-associated secretory phenotype (SASP). Notchdominant secondary senescence relies on expression of growth factors and TGF $\beta$ as well as the induction of fibrillar collagens. SASP-dominant secondary senescence, on the other hand, is driven by release of proinflammatory cytokines, expression of C/EBP $\beta$ and spread of SASP. Both Notch-driven and SASP-driven secretome profiles are found to contribute to a population of secondary senescent cells. Under normal conditions, the combined consequences of paracrine and juxtacrine signalling include the recruitment of immune cells and clearance of senescent cells. Here, primary and secondary senescent cells are viewed as functionally distinct endpoints
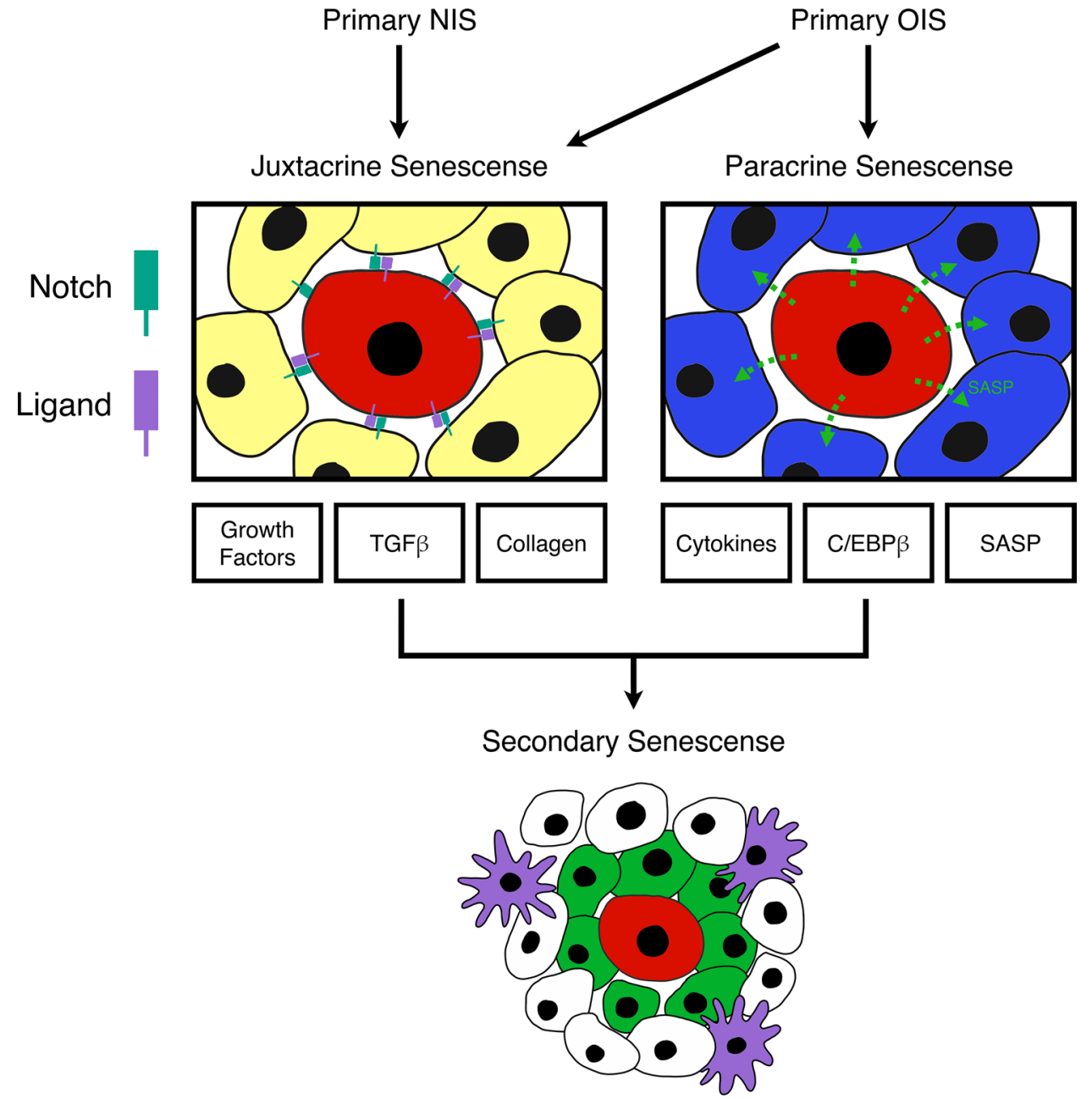

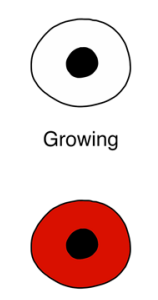

Primary Senescense

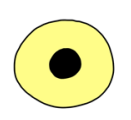

Secondary NIS

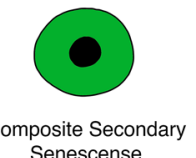

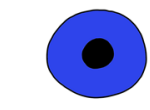

Secondary Paracrine

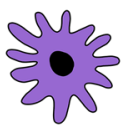

Immune Cell phases is largely understudied, nevertheless. To understand whether and how secondary senescence is shaped by such a complex and dynamic programme, future investigation must include novel approaches that can dissect the relative contribution of each phase towards the induction of secondary senescence as well as determine their interactions with one another. Another tantalising area of study is to test whether the secondary paracrine wave of OIS also holds true in other senescence settings, from replicative senescence to DNA damage-induced senescence. The following question will then be asked: does the absence or presence of secondary senescence serve as a differentiating marker between OIS and other types of senescence, and does it have any physiological implications?

From a biological point of view, a conjectural interpretation of the composite transcriptional NOTCH and SASP signature in secondary senescence represents a fine control of cell proliferation activity and immune surveillance in the senescent milieu. Dysregulation of the initial stage of NOTCH-mediated senescence might promote tissue fibrosis through overexpression of collagen. Likewise, long-term persistence of inflammation or downregulation of NOTCH signalling in the late SASP-rich phase would in turn result in immunosuppression in the cellular neighbourhood and 
decrease the efficiency of immune-clearance of tumourprone senescent cells, thereby favouring pre-neoplastic transformation in normal bystander cells [55]. In this context, alterations or disruption to the dynamic transition from the TGF $\beta$-driven to the C/EBP $\beta$-driven SASP secretomes might explain the dual and pleiotropic role of OIS in cancer formation. Therefore, under normal physiological conditions, the ultimate effects of juxtacrine senescence might lead to a scenario where the spread of senescence within a tissue is limited locally once secondary senescence is established [Fig. 3]. This local containment of senescence spread would guarantee tissue homeostasis and might be beneficial for immune cells to effectively eliminate detrimental senescent cells from that local environment. Verification of this functional model would be assisted by evidence in vitro and in vivo showing that NOTCH-enriched secondary senescent cells represent not a transient but a definitive transcriptional endpoint from which no further senescence is propagated and meaningful and effective immune-clearance can arise. Since primary senescence is widely recognised to suppress tumours, stimulate wound healing and play a role in the ageing process, it will be tantalising to investigate the function of secondary juxtacrine senescence in the contexts of tumour suppression, wound healing and ageing phenotypes in future studies.

\section{Transmission of senescence in organs}

Senescent cell accumulation takes place in many tissues and organs, including the liver and the kidney, often in association with a functional decline and homeostatic capacity of those organs [56]. In hepatocellular senescence, the hepatocytes gradually lose regenerative potential and have increased susceptibility to chronic damage, cirrhosis, fibrosis and hepatocellular carcinoma $[57,58]$.
Compelling evidence suggests that hepatocytes undergoing primary senescence upon acute liver injury in the AhCre-Mdm $2^{\mathrm{f} / / \mathrm{fl}}$ model can spread the senescence phenotype to nearby normal hepatocytes via SASP and the reliance on TGF $\beta$ signalling [52]. The TGF $\beta$-dependent transmission of senescence was also reported in senescent cholangiocytes, which induced secondary senescence in normal surrounding hepatocytes while inhibition of TGF $\beta$ disrupted the transfer of senescence and restored liver function [59]. Combined with our recent in vivo liver data that revealed NIS signature in secondary senescent hepatocytes [54], it is possible that transmission of senescence in the liver is synergistically achieved by both paracrine induction and juxtacrine events.

The kidney is also known to be affected by constituents of SASP [60-62]. A variety of cell types in the kidney such as renal epithelial, endothelial, tubular and glomerular cells undergo age-associated changes over time with simultaneous upregulation of senescence markers [56, 63, 64]. Characteristics of aged kidneys include glomerulosclerosis, interstitial fibrosis and nephron atrophy, which contribute to an increased risk of chronic kidney injury and ultimately acute renal failure $[65,66]$. In a mouse model of renal fibrosis, attenuation of p16 led to a decrease in interstitial fibrosis and nephron atrophy after ischemia-reperfusion injury, suggesting that inhibition of senescence could benefit renal function. This was further shown in transgenic mice where targeted ablation of p16-positive senescent cells attenuated glomerulosclerosis and helped to maintain healthy blood urea nitrogen levels [46]. Mounting evidence has shown that during chronic kidney disease SASP factors are secreted from endothelial cells and macrophages [60]. Consistently, a knockdown of plasminogen activator inhibitor-1 (PAI-1), a SASP factor, attenuated the formation of renal fibrosis upon kidney injury [67]. Interestingly, NOTCH signalling has also been implicated in senescent kidney cells. In mouse based study, the prolonged activation of tubular epithelial

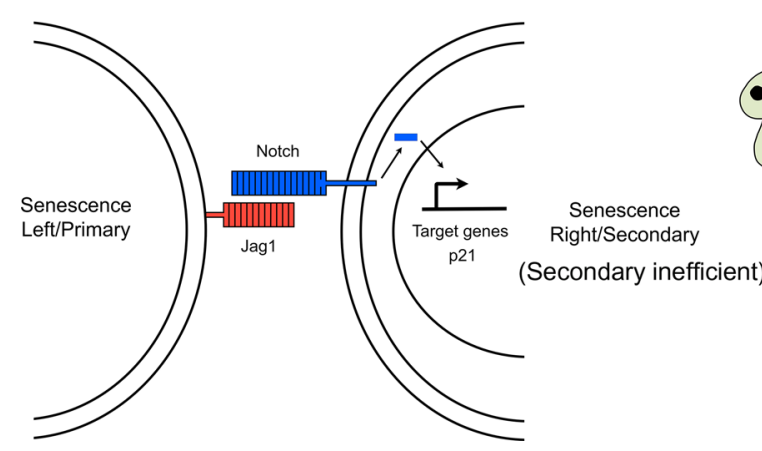

Fig. 3 Schematic representation of the local containment of secondary senescence. Since the Notch ligand JAG1 is upregulated specifically in primary senescence cells, it could be possible that the secondary spread of senescent cells can be contained locally, as opposed to the senescence process expanding endlessly once established. Sec- ondary senescent cells, which express low levels of the ligand JAG1, will not be able to induce further senescence responses. Such a local containment might allow unwanted senescent cells to be efficiently eliminated by the immune system 
NOTCH1 diminished the regenerative potential of the kidney and was associated with the pro-senescent phenotype [68]. Yet not much is known about the establishment of secondary senescence by juxtacrine NOTCH signalling in the context of the senescent kidney. It will be useful and important to investigate the transcriptomes of senescent kidney cells and determine if primary and secondary senescence signatures can be distinguished and to what extent NOTCH and SASP contribute to secondary senescence.

\section{Concluding remarks}

Oncogene-induced senescence unmistakably represents a tumour suppressor mechanism, but our understanding of its induction and establishment is not yet complete. The heterogeneity of the OIS programme is only beginning to be elucidated through the use of single-cell technologies. Among the key questions that need to be addressed are whether all cells respond similarly to the same oncogenic activation and whether the same oncogenic insult always results in a heterogenous OIS population. We have summarised current evidence for a functional heterogeneity in the senescence response in a recent review [69]. Studies of OIS cells and their transcriptomes in vitro and in vivo have unmasked a complex regulation of their dynamic and heterogeneous nature. Rather than being a stable entity following its triggers, OIS is endowed with both autonomous and non-cell autonomous activities, which allow OIS cells to interact with their neighbouring normal or transformed cells. One major effect of this interaction is the paracrine transmission of the senescent phenotype, largely mediated by secretion of a vast array of SASP factors, contributing to a secondary induction of senescence in the surrounding environment.

OIS, primary or secondary, is traditionally believed to have only one functional endpoint, but recent research has favoured the notion that primary and secondary senescent cells are in fact functionally distinct stages of OIS. Furthermore, transcriptomic analyses have challenged the prevailing view that paracrine induction of senescence is the only main mediator of secondary senescence in OIS, with juxtacrine NOTCH signalling emerging as a synergistic driver of secondary senescence. This means that OIS is a far more complex state than previously anticipated, with autocrine, paracrine and juxtacrine signalling pathways being at play. The precise mechanisms that govern the induction of senescence through paracrine or juxtacrine signalling, however, remain elusive. It is highly possible that the secondary induction of OIS is dictated by the dynamically spatiotemporal patterns of the senescence-associated secretomes as well as cellto-cell interactions, which in turn shape the varied quality, quantity, output and changes of OIS functionality. To obtain a better insight into the molecular basis for the regulation of secondary senescence, novel technologies such as single-cell transcriptomics and genomics will prove valuable in identifying and characterising more subtle events taking place over the course of OIS, primary or secondary. Accordingly, investigating the heterogeneity of a senescent cell population is one approach to uncovering the actual contribution of each mode of senescence transmission, and will significantly highlight directions for the strategic development of new and specific drugs that target deleterious senescent cells.

Open Access This article is licensed under a Creative Commons Attribution 4.0 International License, which permits use, sharing, adaptation, distribution and reproduction in any medium or format, as long as you give appropriate credit to the original author(s) and the source, provide a link to the Creative Commons licence, and indicate if changes were made. The images or other third party material in this article are included in the article's Creative Commons licence, unless indicated otherwise in a credit line to the material. If material is not included in the article's Creative Commons licence and your intended use is not permitted by statutory regulation or exceeds the permitted use, you will need to obtain permission directly from the copyright holder. To view a copy of this licence, visit http://creativecommons.org/licenses/by/4.0/.

\section{References}

1. Kuilman T, Michaloglou C, Mooi WJ, Peeper DS (2010) The essence of senescence. Genes Dev 24:2463-2479

2. Campisi J (2013) Aging, cellular senescence, and cancer. Annu Rev Physiol 75:685-705

3. van Deursen JM (2014) The role of senescent cells in ageing. Nature 509:439-446

4. Hayflick L, Moorhead PS (1961) The serial cultivation of human diploid cell strains. Exp Cell Res 25:585-621

5. Harley CB, Futcher AB, Greider CW (1990) Telomeres shorten during ageing of human fibroblasts. Nature 345:458-460

6. He S, Sharpless NE (2017) Senescence in health and disease. Cell 169:1000-1011

7. Herbig U, Jobling WA, Chen BPC, Chen DJ, Sedivy JM (2004) Telomere shortening triggers senescence of human cells through a pathway involving ATM, p53, and p21CIP1, but not p16INK4a. Mol Cell 14:501-513

8. di Fagagna d'Adda F et al (2003) A DNA damage checkpoint response in telomere-initiated senescence. Nature 426:194-198

9. di Fagagna d'Adda F (2008) Living on a break: cellular senescence as a DNA-damage response. Nat Rev Cancer 8:512-522

10. Dimri GP et al (1995) A biomarker that identifies senescent human cells in culture and in aging skin in vivo. Proc Natl Acad Sci U S A 92:9363-9367

11. Coppé J-P et al (2008) Senescence-associated secretory phenotypes reveal cell-nonautonomous functions of oncogenic RAS and the p53 tumor suppressor. PLoS Biol 6:e301

12. Georgakopoulou EA et al (2013) Specific lipofuscin staining as a novel biomarker to detect replicative and stress-induced senescence. A method applicable in cryo-preserved and archival tissues. Aging (Albany. NY) 5:37-50

13. Myrianthopoulos V et al (2019) Senescence and senotherapeutics: a new field in cancer therapy. Pharmacol Ther 193:31-49

14. Evangelou $\mathrm{K}$ et al (2017) Robust, universal biomarker assay to detect senescent cells in biological specimens. Aging Cell 16:192-197 
15. Gorgoulis V et al (2019) Cellular senescence: defining a path forward. Cell 179:813-827

16. Sarkisian CJ et al (2007) Dose-dependent oncogene-induced senescence in vivo and its evasion during mammary tumorigenesis. Nat Cell Biol 9:493-505

17. Kamijo T et al (1997) Tumor suppression at the mouse INK4a locus mediated by the alternative reading frame product $\mathrm{p} 19 \mathrm{ARF}$. Cell 91:649-659

18. Ventura A et al (2007) Restoration of p53 function leads to tumour regression in vivo. Nature 445:661-665

19. Xue W et al (2007) Senescence and tumour clearance is triggered by p53 restoration in murine liver carcinomas. Nature 445:656-660

20. Rayess H, Wang MB, Srivatsan ES (2012) Cellular senescence and tumor suppressor gene p16. Int J Cancer 130:1715-1725

21. Chicas A et al (2010) Dissecting the unique role of the retinoblastoma tumor suppressor during cellular senescence. Cancer Cell 17:376-387

22. Zhuang D et al (2008) C-MYC overexpression is required for continuous suppression of oncogene-induced senescence in melanoma cells. Oncogene 27:6623-6634

23. Denoyelle C et al (2006) Anti-oncogenic role of the endoplasmic reticulum differentially activated by mutations in the MAPK pathway. Nat Cell Biol 8:1053-1063

24. Cipriano R et al (2011) TGF-beta signaling engages an ATMCHK2-p53-independent RAS-induced senescence and prevents malignant transformation in human mammary epithelial cells. Proc Natl Acad Sci U S A 108:8668-8673

25. Couso JP, Bishop SA, Martinez Arias A (1994) The wingless signalling pathway and the patterning of the wing margin in Drosophila. Development 120:621-636

26. Hoare $\mathrm{M}$ et al (2016) NOTCH1 mediates a switch between two distinct secretomes during senescence. Nat Cell Biol 18:979-992

27. Parry AJ et al (2018) NOTCH-mediated non-cell autonomous regulation of chromatin structure during senescence. Nat Commun 9:1840

28. Collado $M$ et al (2005) Senescence in premalignant tumours. Nature 436:642-642

29. Braig $\mathrm{M}$ et al (2005) Oncogene-induced senescence as an initial barrier in lymphoma development. Nature 436:660-665

30. Chen Z et al (2005) Crucial role of p53-dependent cellular senescence in suppression of Pten-deficient tumorigenesis. Nature 436:725-730

31. Michaloglou C et al (2005) BRAFE600-associated senescencelike cell cycle arrest of human naevi. Nature 436:720-724

32. Pollock PM et al (2003) High frequency of BRAF mutations in nevi. Nat Genet 33:19-20

33. Curtin JA et al (2005) Distinct sets of genetic alterations in melanoma. N Engl J Med 353:2135-2147

34. Liu JY et al (2019) Cells exhibiting strong p16 INK4a promoter activation in vivo display features of senescence. Proc Natl Acad Sci U S A 116:2603-2611

35. Bianchi-Smiraglia A, Lipchick BC, Nikiforov MA (2017) The immortal senescence. Methods Mol Biol 1534:1-15

36. Acosta JC et al (2008) Chemokine signaling via the CXCR2 receptor reinforces senescence. Cell 133:1006-1018

37. Kuilman $\mathrm{T}$ et al (2008) Oncogene-induced senescence relayed by an interleukin-dependent inflammatory network. Cell 133:1019-1031

38. Acosta JC et al (2013) A complex secretory program orchestrated by the inflammasome controls paracrine senescence. Nat Cell Biol 15:978-990

39. Hubackova S, Krejcikova K, Bartek J, Hodny Z (2012) IL1and TGF $\beta$-Nox 4 signaling, oxidative stress and DNA damage response are shared features of replicative, oncogene-induced, and drug-induced paracrine 'Bystander senescence'. Aging (Albany. NY) 4:932-951

40. Lesina $\mathrm{M}$ et al (2016) RelA regulates CXCL1/CXCR2-dependent oncogene-induced senescence in murine Kras-driven pancreatic carcinogenesis. J Clin Invest 126:2919-2932

41. Iannello A, Thompson TW, Ardolino M, Lowe SW, Raulet DH (2013) p53-dependent chemokine production by senescent tumor cells supports NKG2D-dependent tumor elimination by natural killer cells. J Exp Med 210:2057-2069

42. Malaquin $\mathrm{N}$ et al (2013) Senescent fibroblasts enhance early skin carcinogenic events via a paracrine MMP-PAR-1 axis. PLoS ONE 8:e63607

43. Laberge R-M et al (2015) MTOR regulates the pro-tumorigenic senescence-associated secretory phenotype by promoting IL1A translation. Nat Cell Biol 17:1049-1061

44. Eggert T et al (2016) Distinct functions of senescence-associated immune responses in liver tumor surveillance and tumor progression. Cancer Cell 30:533-547

45. Baker DJ et al (2011) Clearance of p16Ink4a-positive senescent cells delays ageing-associated disorders. Nature 479:232-236

46. Baker DJ et al (2016) Naturally occurring p16Ink4a-positive cells shorten healthy lifespan. Nature 530:184-189

47. Grosse L, Wagner N, Emelyanov A et al (2020) Defined p16High senescent cell types are indispensable for mouse healthspan. Cell Metab 32(1):87-99.e6

48. Gomis RR, Alarcón C, Nadal C, Van Poznak C, Massagué J (2006) $\mathrm{C} / \mathrm{EBP} \beta$ at the core of the TGF $\beta$ cytostatic response and its evasion in metastatic breast cancer cells. Cancer Cell 10:203-214

49. Mosteiro L, Pantoja C, de Martino A, Serrano M (2018) Senescence promotes in vivo reprogramming through p16INK4a and IL-6. Aging Cell 17(2):e12711

50. Ritschka B et al (2017) The senescence-associated secretory phenotype induces cellular plasticity and tissue regeneration. Genes Dev 31:172-183

51. Biran A et al (2014) Senescent cells communicate via intercellular protein transfer. Genes Dev 29:791-802

52. Takasugi M, Okada R, Takahashi A, Virya Chen D, Watanabe S, Hara E (2017) Small extracellular vesicles secreted from senescent cells promote cancer cell proliferation through EphA2. Nat Commun 8:15729

53. Borghesan M et al (2019) Small extracellular vesicles are key regulators of non-cell autonomous intercellular communication in senescence via the interferon protein IFITM3. Cell Rep 27:39563971.e6

54. Teo YV et al (2019) Notch signaling mediates secondary senescence. Cell Rep 27:997-1007.e5

55. Lee S, Schmitt CA (2019) The dynamic nature of senescence in cancer. Nat Cell Biol 21:94-101

56. Sturmlechner I, Durik M, Sieben CJ, Baker DJ, Van Deursen JM (2017) Cellular senescence in renal ageing and disease. Nat Rev Nephrol 13:77-89

57. Rudolph KL, Chang S, Millard M, Schreiber-Agus N, DePinho RA (2000) Inhibition of experimental liver cirrhosis in mice by telomerase gene delivery. Science (80-.) 287:1253-1258

58. Cieslak KP, Baur O, Verheij J, Bennink RJ, van Gulik TM (2016) Liver function declines with increased age. HPB 18:691-696

59. Ferreira-Gonzalez S, Lu WY, Raven A et al (2018) Paracrine cellular senescence exacerbates biliary injury and impairs regeneration. Nat Commun 9(1):1020

60. Prattichizzo F et al (2018) Short-term sustained hyperglycaemia fosters an archetypal senescence-associated secretory phenotype in endothelial cells and macrophages. Redox Biol 15:170-181

61. Zhang Y et al (2017) A new role for oxidative stress in aging: the accelerated aging phenotype in Sod1-/- mice is correlated to increased cellular senescence. Redox Biol 11:30-37 
62. Knoppert SN, Valentijn FA, Nguyen TQ, Goldschmeding R, Falke LL (2019) Cellular senescence and the kidney: potential therapeutic targets and tools. Front Pharmacol 10

63. Darmady EM, Offer J, Woodhouse MA (1973) The parameters of the ageing kidney. J Pathol 109:195-207

64. Rule AD et al (2010) The association between age and nephrosclerosis on renal biopsy among healthy adults. Ann Intern Med 152:561-567

65. Megyesi J, Safirstein RL, Price PM (1998) Induction of p21(WAF1/CIP1/SD11) in kidney tubule cells affects the course of cisplatin-induced acute renal failure. J Clin Invest 101:777-782

66. Zhou $\mathrm{H}$ et al (2004) The induction of cell cycle regulatory and DNA repair proteins in cisplatin-induced acute renal failure. Toxicol Appl Pharmacol 200:111-120

67. Yao L et al (2019) Fibroblast-specific plasminogen activator inhibitor-1 depletion ameliorates renal interstitial fibrosis after unilateral ureteral obstruction. Nephrol Dial Transplant. https:// doi.org/10.1093/ndt/gfz050

68. Sörensen-Zender I et al (2014) Renal tubular Notch signaling triggers a prosenescent state after acute kidney injury. Am J Physiol Physiol 306:F907-F915

69. Kirschner K, Rattanavirotkul N, Quince MF, Chandra T (2020) Functional heterogeneity in senescence. Biochem Soc Trans 48:765-773

Publisher's Note Springer Nature remains neutral with regard to jurisdictional claims in published maps and institutional affiliations. 